\title{
Assessing the effect of RET transmembrane domain mutations in receptor self-association capability using the in vivo TOXCAT system
}

\author{
M. BENEJ ${ }^{1}$, S. FEKECSOVA ${ }^{2}$, M. POTURNAJOVA ${ }^{3, *}$ \\ ${ }^{1}$ Institute of Virology SAS, Dubravska cesta 9, 84505 Bratislava; ${ }^{2}$ Faculty of Natural Sciences, Commenius University, Mlynska dolina, 84215 Bra- \\ tislava; ${ }^{3}$ Cancer Research Institute SAS, Vlarska 7, 83391 Bratislava
}

${ }^{*}$ Correspondence: martina.poturnajova@savba.sk

Received June 8, 2012/ Accepted September 13, 2012

\begin{abstract}
Mutations of $c$-RET proto-oncogene with a unique localization within the human transmembrane receptor represent a challenge for contemporary molecular oncology techniques. RET transmembrane domain (TMD)-driven dimerization of the receptor leads to its permanent activation that eventually results in the development of medullary thyroid neoplasia. In this study, we describe the employment of the TOXCAT system which enables to investigate mutationinduced alterations in the strength of RET TMD dimerization in vivo. We suggest an improvement of the method by adding reporter gene quantification at the mRNA levels as a support to the commonly used reporter protein level. We have investigated possible changes in RET TMD dimerization in case of two germline RET TMD mutations found in in several individual cases and MEN2 families worldwide, p.Ala641Ser and p.Ser649Leu. According to our results, substitution of Ser-649 residue by leucine, found as a result of germline mutation, caused a significant decrease of RET TMD self-association in comparison to RET wild-type transmembrane domain. The impaired ability of self-association suggests a novel, yet unknown mechanism of tyrosine kinase domain activation, possibly independent of RET homodimerization.
\end{abstract}

Key words: RET mutation, transmembrane domain, molecular oncology, TOXCAT system, oncoprotein dimerization

Medullary thyroid carcinoma (MTC) is a tumor that arises from parafollicular C cells located in the thyroid gland. Approximately $5-8 \%$ of all thyroid malignancies are represented by MTC [1]. The majority of MTC cases are sporadic, whereas $20-30 \%$ belong to the hereditary form [2]. Hereditary MTC is the most prevalent, hence obligatory manifestation of Multiple endocrine neoplasia type 2 (MEN 2) syndrome. The incidence of autosomal-dominant hereditary MEN 2 syndrome reaches 2.5 cases in 100000 in general population [3]. MEN 2 syndrome is clinically divided into three distinct subtypes varying in the age of onset, rate of incidence, molecular-genetic mechanisms of onset and aggressiveness of MTC: MEN 2A, MEN 2B and familial MTC [4-6].

Except for rare cases, MTC is triggered by germline mutations of a single allele of $c-R E T$, a cellular proto-oncogene [7-9]. The gene encodes RET tyrosine kinase receptor, involved in signal transduction responsible for growth and differentiation in early stages of embryogenesis [10]. Mutations of the $c-R E T$ gene are divided into four risk levels, $\mathrm{A}-\mathrm{D}$, with the
D level being the highest risk level [11]. The rationale for this classification lies in association of distinct mutations with specific aggressiveness of the MTC phenotype. Another classification is based on the position of specific mutation relative to the plasma membrane [12]. In both of these classifications, mutations of the transmembrane domain of RET protein are considered as a "low-risk" category.

In most cases, the oncogenic transformation of $c-R E T$ is triggered by mutation-induced homodimerization of the RET receptor and by covalent linkage of the RET homodimer by disulfide bonds between unpaired cysteine residues. This leads to permanent activation of downstream signaling pathways. Kjaer et al. proposed a role of the transmembrane domain (TMD) of the RET protein as a potential mediator of RET protein homodimerization [13]. The basis of this assumption lies in determination of the capability of RET TMD self-association. RET TMD is formed by a single hydrophobic $\alpha$-helix. Three amino acid residues responsible for TMD self-association have been identified: Ser-649, Ser-653 and indirectly Ala-641 [13]. 


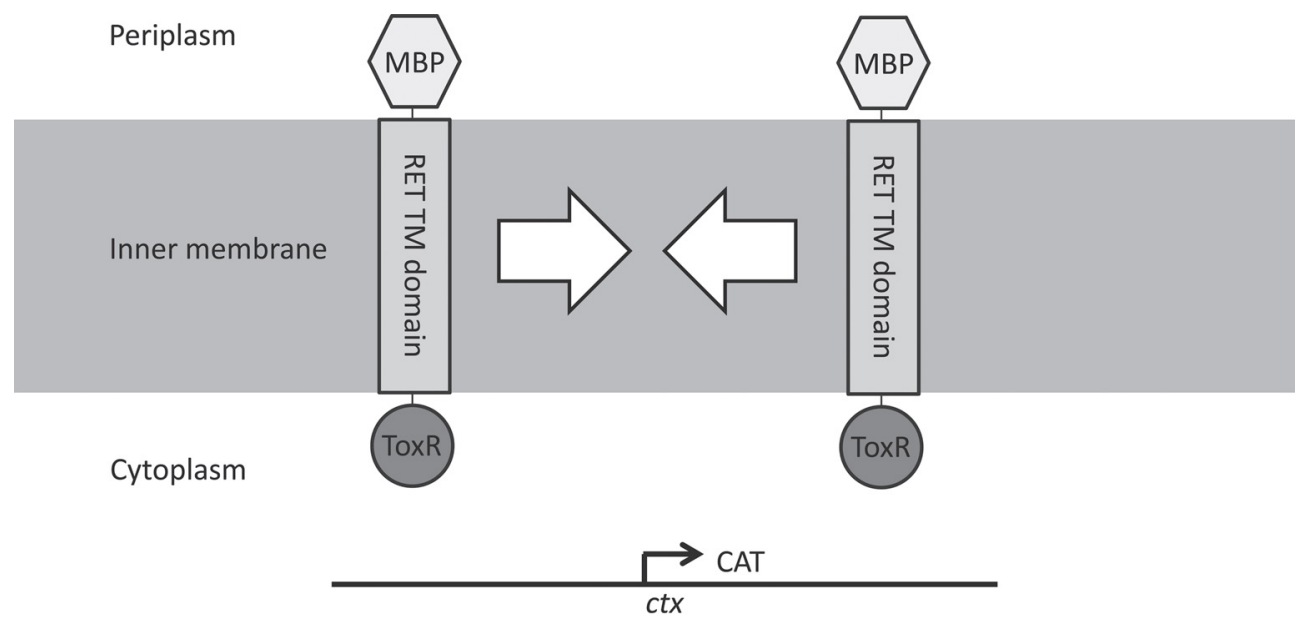

Figure 1. Schematic representation of ToxR-RET TMD-MBP fusion proteins. C-terminal domain is located in the periplasm and represented by maltose binding protein (MBP). The body of the protein is formed by cloned RET transmembrane domain (TMD), and the N-terminal domain is impersonated by ToxR, a dimerization-dependent transcription activator. TMD-triggered dimerization of the fusion proteins results in ToxR dimerization, which subsequently activates the ctx promoter and induces CAT reporter gene expression. The strength of dimerization is equal to the rate of reporter gene expression.

According to the previous conclusions made by Kjaer et al., RET TMD may serve as the "driving force" that brings two mutant RET molecules to a proximity required for disulfide bond creation.

The molecular-genetic mechanisms by which mutations in TMD activate the $c$-RET oncogene have not been elucidated yet [14]. Analyzing the strength of RET TMD dimerization in case of specific TMD mutations might shed more light into this issue.

Thus, a method enabling to determine mutation-induced alterations in the strength of RET TMD was required. Among possible denaturing and non-denaturing methods serving this purpose, TOXCAT system introduced by Russ \& Engelman was selected as the most appropriate [15]. This in vivo approach provides an environment of natural phospholipid bilayer for TMD self-association analyses. The method represents an enhancement of ToxR dimerization assay which was introduced in 1996 by Langosch et al [16]. The principle of TOXCAT system lies in TMD-driven dimerization of ToxR, a dimerization-dependent transcription activator. Dimerized ToxR binds to cholera toxin gene (ctx) promoter, thereby inducing expression of the reporter gene - chloramphenicol acetyltransferase (CAT). CAT expression rate is then equal to the extent of TMD self-association. ToxR dimerization is achieved by fusing the tested TMD to ToxR. The final fusion protein includes maltose binding protein (MBP, or MalE) as well, to allow delivery to the E. coli inner membrane (Figure 1). Thus, the TMD region of the MBP-TMD-ToxR fusion protein is allowed to interact within the environment of a biological membrane, providing the rationale of our understanding of RET TMD self-association.

This study utilizes the TOXCAT system to examine the possible role of TMD self-association as an activating effect in case of two RET TMD mutations. This hypothesis is based on the fact that these mutations were detected in MEN2A positive members of two Slovak families and affect two of the three amino acid residues mediating RET TMD self-association, p.Ala641Ser (GCT-> TCT) and p.Ser649Leu (TCG->TTG). The latter belongs to rare RET TMD mutations occurring as a double mutation in combination with other mutations or as a sole mutation in several MTC positive individuals throughout the world. However, the molecular mechanism, by which this mutation activates RET has not been elucidated [17-19]. The aim is to gain evidence required for better understanding of genotype: phenotype correlation in case of these two mutations, as well as to examine the possibility of establishing TOXCAT system for TMD interaction analyses in molecular oncology.

\section{Material and methods}

Preparation of p.Ala641Ser and p.Ser649Leu homozygous variants. Frozen MTC tissue of patient with p.Ala641Ser mutation was used for cDNA synthesis according to the standard protocol for reverse transcription. The coding sequence of full-length c- $R E T$ gene $3376 \mathrm{bp}$ was amplified in two nested PCR reactions using a mixture of proofreading and robust DNA polymerases (Long PCR enzyme mix, Thermo Fisher). PCR product was inserted into bacterial vector pTZ57R/T using TA cloning (InsTAclone ${ }^{\mathrm{m}}$ PCR Cloning Kit, Thermo Fisher) and transformed into E.coli DH5a competent cells. The construct carrying p.Ala641Ser mutation in fulllength c-RET in correct orientation was verified and selected by ampicilin selection, enzyme digestions, PCR detection of insert and direct sequencing.

In vitro oligonucleotide site-directed mutagenesis system with specific primers was used for construction of clones 
either without p.Ala641Ser (wild-type) and, subsequently, with p.Ser649Leu introduced mutations in the c-RET gene (QuikChange Site-Directed Mutagenesis Kit, Stratagene). Constructs were verified by direct sequencing.

Preparation of TOXCAT constructs. Regions of wild-type RET TMD (codons 635-657) as well as of RET TMDs carrying p.Ala641Ser and p.Ser649Leu mutations were amplified from healthy blood donor and pTZ57R+mutRET, respectively, using the primers TOX KJ S+A that add BamHI and NheI restriction sites to the termini of the amplicons (Table 1). The amplicons, as well as pccKAN plasmid vector (kindly provided by Prof. Engelman, Yale, USA, designed by Russ \& Engelman [15]) were digested using NheI and BamHI restriction enzymes. Ligation was performed using T4 DNA Ligase (Thermo Fisher) in 1:5 and 1:20 vector: insert ratios, creating RET wt-pccKAN, Ala641Ser-pccKAN and Ser649Leu-pccKAN TOXCAT constructs.

Plasmid DNA minipreps were screened for the presence of RET TMD by real-time PCR assay using the RET TM S+A primers (Table 1). TOXCAT constructs with detected TMD were transformed into E. coli NT326 cells by electroporation. In addition, positive control plasmids ToxR-GpAwt-MBP and ToxR-GpA G83I-MBP (kind gift of Prof. Engelman, Yale University, USA), used for verification of TOXCAT method, were transformed into E. coli NT326 cells by electroporation.

Maltose complementation assay. Single bacterial colonies of E.coli NT326 carrying TOXCAT constructs were cultivated in $5 \mathrm{ml}$ of liquid LBA medium until $\mathrm{OD}_{600} \sim 0.5-0.6$. One loop of each bacterial culture was streaked on solid M9 minimal medium containing maltose as the only source of carbon and incubated at $37^{\circ} \mathrm{C}$ for 96 hours. As negative controls, untransformed E. coli NT326 bacteria and NT326 bacteria transformed with digested and re-ligated pccKAN vector were used. The viability of all clones was confirmed by performing the same experiment using solid M9 medium containing glucose as a carbon source. M9 minimal medium was prepared according to Ausubel et al. [20].

Disk Diffusion assay. LBA plates were pre-incubated overnight at $37^{\circ} \mathrm{C}$ with a Whatman filter paper disk $(28 \mathrm{~mm}$ diameter, in the centre of the Petri dish) containing $120 \mu$ of chloramphenicol $(61 \mathrm{mg} / \mathrm{ml})$. Single bacterial colonies were cultivated in $5 \mathrm{ml}$ of liquid LBA medium until $\mathrm{OD}_{600} \sim 0.4-0.5$, $100 \mu$ of this culture was mixed $1: 1$ with $\mathrm{mQ}_{2} 0$ and spread on LBA plates with chloramphenicol and incubated overnight at $37^{\circ} \mathrm{C}$. The diameter of the zone of inhibition (ZOI) circumscribing the disk was measured. All bacterial clones were analyzed in triplicates and the ZOI size was measured 10 times.

\section{qPCR CAT Assay.}

cDNA Synthesis. Overnight culture of specific TOXCAT constructs in NT326 bacteria were inoculated in $2 \mathrm{ml}$ of liquid LBA medium and grown until log phase $\left(\mathrm{OD}_{600} \sim 0.4\right.$ $0.5)$. Total RNA was isolated using NucleoSpin ${ }^{\circ}$ RNA II Kit (Macherey-Nagel) and DNA traces were removed by RNasefree DNaseI treatment. Total RNA was verified by PCR for complete DNA removal and then reverse transcribed into cDNA using RevertAid ${ }^{\mathrm{mm}} \mathrm{H}$ Minus First Strand cDNA Synthesis Kit (Thermo Fisher). Quantity and quality of purified cDNA were determined using spectrophotometry.

Housekeeping Gene Protocol. 16S ribosomal RNA gene was chosen as housekeeping gene for expression analysis in E.coli [21-22] using 16S rRNA "universal" primers 357F and 519R [23] (Table 1). Quantitative PCR was performed using 2x Maxima ${ }^{\circledR}$ Probe qPCR Master Mix (Thermo Fisher).

Reporter Gene Protocol. Reporter gene primers and probe, CAT2 S+A, and CAT2 FAM dual-labeled probe, respectively, were designed using Vector NTI software (Invitrogen) according to transposon Tn9 chloramphenicol acetyltransferase gene (GenBank ID: U46780.1) (Table 1). The amplification was performed using Maxima ${ }^{\circ}$ Probe qPCR Master Mix (Thermo Fisher). Correct amplification of samples was verified on $1 \%$ agarose gel. Plasmids carrying cDNA of positive control clones (ToxR-GpAwt-MBP and ToxR-GpA G83I-MBP) were used for validation.

Statistical Analysis and Validation. Normalized fold expression for each sample relative to negative control was determined by $\Delta \Delta \mathrm{Ct}$ method using CFX Manager software 1.5 (Bio-Rad). Resulting expression data for each dilution were exported and processed by Excel 2007 (Microsoft) and normalized to RET wild-type sample. To validate the signifi-

Table 1. Sequences the primer pairs used in the study.

\begin{tabular}{|c|c|}
\hline Primer & Sequence \\
\hline TOX KJ S & $5^{\prime}$ - CCGCAG $\frac{\text { NCTAGCGGCCGCACGGTGATCG - 3' }}{\text { NheI site }}$ \\
\hline TOX KJ A & $5^{\prime}$ - GGCGCAAT $\underset{\text { Bam HI site }}{\text { Bam CCGATGCAGAAGGCAGACAG - } 3^{\prime}}$ \\
\hline RET TM S & $5^{\prime}$ - CGCACGGTGATCGCAGCC - 3' \\
\hline RET TM A & $5^{\prime}$ - GATGCAGAAGGCAGACAGC - 3' \\
\hline $357 \mathrm{~F}$ & $5^{\prime}$ - CTCCTACGGGAGGCAGCAG - 3’' \\
\hline $519 \mathrm{R}$ & $5^{\prime}$ - GAATTACCGCGGCTGCTG - $3^{\prime}$ \\
\hline CAT $2 \mathrm{~S}$ & $5^{\prime}$ - CCGGCCTTTATTCACATTCTTGC - 3' \\
\hline CAT 2 A & $5^{\prime}$ - GGAAAACGGTGTAACAAGGGTGAA - 3' \\
\hline CAT 2 probe & 5’ - FAM-AATGCTCATCCGGAATTCCGTATGGCAATG-BHQ1- 3` \\
\hline
\end{tabular}




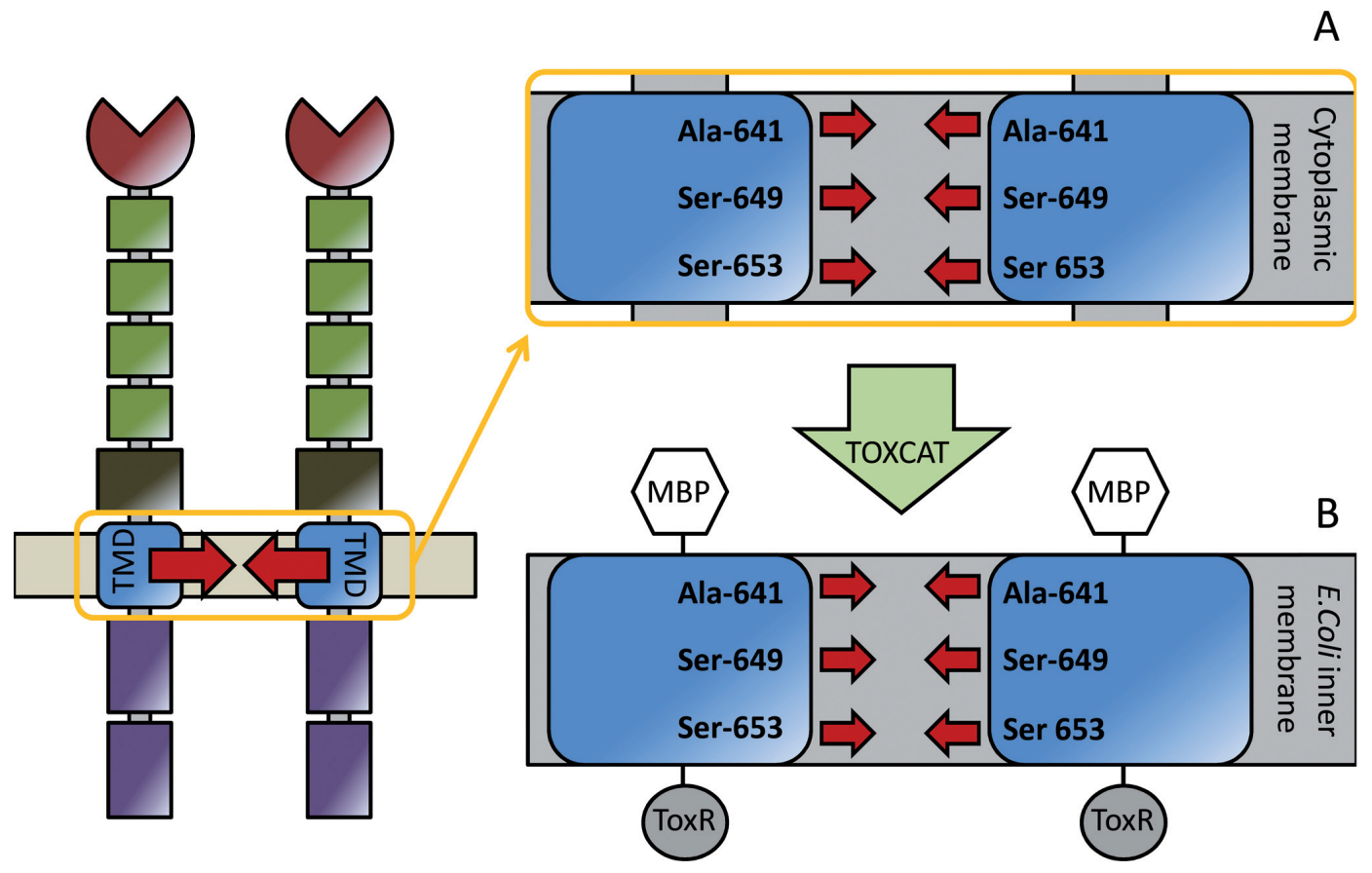

Figure 2. Creation of the fusion proteins. The strength of RET TMD oligomerization, driven by three crucial amino acid residues, Ala-641, Ser-649 and Ser-653 (A) is analyzed by fusion of the RET TMD between MBP protein and ToxR transcription activator. The TOXCAT system (B) provides environment of the E.coli inner memrane to study the interaction occuring between TMDs in the plasma membrane.

cance of the observed expression variations, sample to sample Student's T-test was performed. To analyze overall significance of the data, Analysis of variance (ANOVA) statistical model was utilized.

\section{ELISA CAT Assay}

Preparation of protein lysates by enzymatic treatment. Overnight culture of specific TOXCAT construct in NT326 bacteria were inoculated in $10 \mathrm{ml}$ of liquid LBA medium and cultivated until $\mathrm{OD}_{600} \sim 0.4-0.5$. The cultures were pelleted by centrifugation and the pellets were washed twice in ice-cold PBS. Washed pellets were resuspended in $1 \mathrm{ml}$ of TE (50 $\mathrm{mM}$ Tris, $10 \mathrm{mM}$ EDTA, $\mathrm{pH}$ 8) solution containing $0.2 \mathrm{mg} / \mathrm{ml}$ lysozyme, $10 \mathrm{ng} / \mathrm{ml}$ aprotinine, $4 \mathrm{ng} / \mathrm{ml}$ leupeptine, $1 \mathrm{umol} / \mathrm{ml}$ PMSF and $0.1 \mathrm{U} / \mathrm{ml}$ DNase I for lysis and protease inhibition. The mixture was incubated at room temperature for 1 hour, then pelleted by high-speed centrifugation and supernatant was stored at $-20^{\circ} \mathrm{C}$. Total protein concentration of each lysate was measured by standard Lowry method.

ELISA CAT Assay (Roche). Quantification of CAT reporter protein was performed according to manufacturer instructions, with modifications required for adjusting to bacterial CAT reporter system. Briefly, $200 \mu \mathrm{l}$ of CAT enzyme standards or bacterial lysates in parallels were incubated in 96-well plate for 2 hours. Unadhered lysate was washed away and the plate was further incubated with antiCAT-digoxigenin (DIG)-labeled antibody for 1 hour. After additional wash, antiDIG antibody conjugated to peroxidase was added for
1 hour, washed out and detected with ABTS substrate. All incubations were performed at $37^{\circ} \mathrm{C}$. Optical density of each sample was measured at 405 and $490 \mathrm{~nm}$ and the difference of $\mathrm{OD}_{405 \mathrm{~nm}}-\mathrm{OD}_{490 \mathrm{~nm}}$ was determined. Concentration of samples was calculated using the standard curve of CAT enzyme standards. CAT level was determined as pg of CAT protein per $1 \mu \mathrm{g}$ of total proteins in protein lysate. CAT protein levels were validated on qualitative basis by Immunoblots, using monoclonal IgG antiCAT antibody (LS/C56003/25877, LifeSpan Biosciences)(Figure 6D).

\section{Results}

We prepared TOXCAT constructs expressing either ToxR-Ser649Leu-MBP, ToxR-Ala641Ser-MBP or ToxRRETwt-MBP proteins (Figure 2). The capability of TMD self-association manifested by dimerization of the respective fusion protein (thus triggering CAT reporter gene expression) within the inner membrane of E.coli NT326 was analyzed on mRNA and protein level. Obtained CAT gene expression levels, reflecting the strength of TMD self-association were normalized relative to the levels of RET wild type TMD in order to determine mutation-induced changes in RET TMD self-association.

In silico prediction of phenotypic impact. Possible phenotypic impacts of p.Ala641Ser and p.Ser649Leu mutations were predicted using Polymorphism Phenotyping (PolyPhen) 


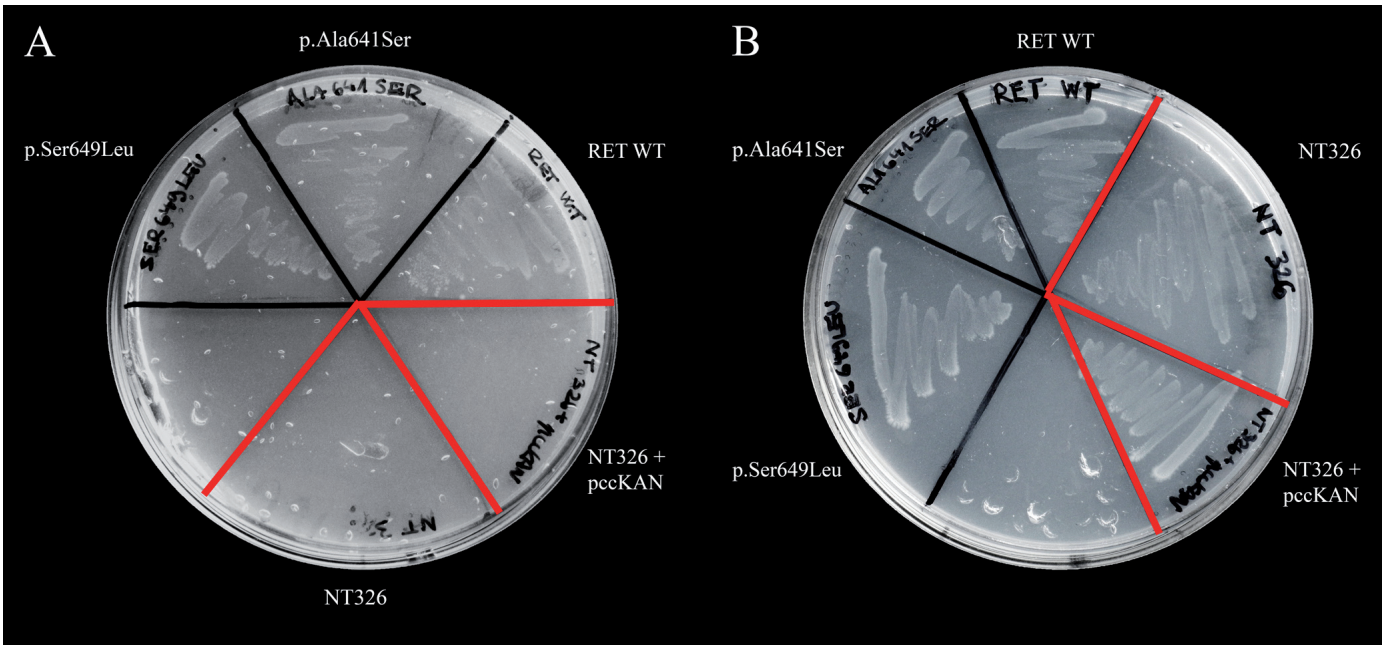

Figure 3. Maltose complementation assay. MBP-deficient E.coli NT326 producing fusion proteins are grown on M9 minimal medium providing maltose as the sole carbon source. Only clones able to complement the MBP-deficient phenotype with the C-terminal domain of ToxR-RET TMD-MBP fusion proteins are able to grow on this medium. Figure (A) represents E.coli NT326 clones grown on M9 minimal medium; figure (B) represents E.coli NT326 clones grown on M9 minimal medium containing glucose as carbon source. Negative controls, represented by untransformed E.coli NT326 competent bacteria and E.coli NT326 bacteria transformed with digested and re-ligated pccKAN, not expressing fusion proteins are highlighted in red.

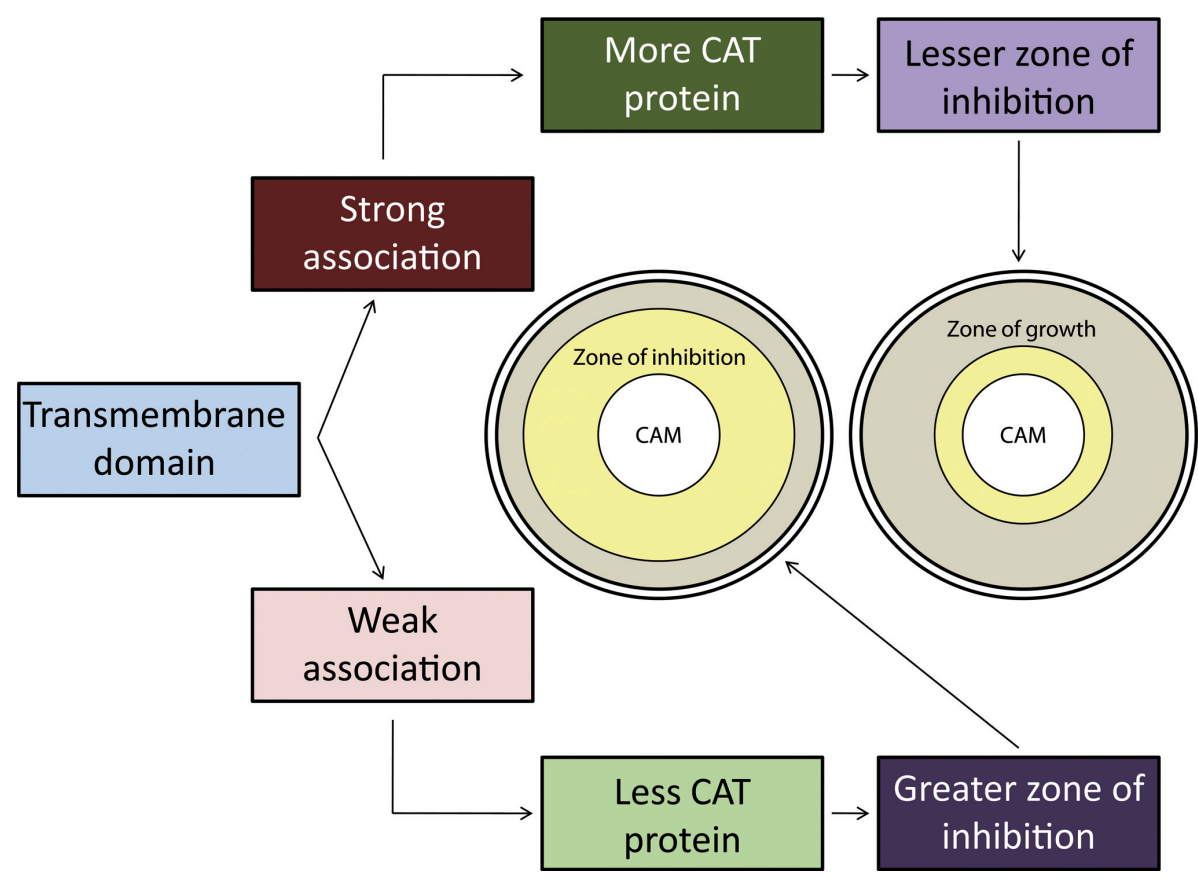

Figure 4. Disk diffusion assay principle. In case of strong transmembrane domain-mediated association of ToxR-TMD-MBP fusion proteins, a greater amount of CAT protein is produced, providing resistance to chloramphenicol (CAM), resulting in lesser zone of inhibition (depicted on schematic Petri dish). In contrary, weaker association is manifested by a smaller amount of CAT protein, resulting in greater zone of inhibition.

mutation prediction tool [24]. This tool predicts possible impact of amino acid substitutions on the structure and function of a distinct protein. Based on sequence annotations, the mutation p.Ala641Ser was predicted to be benign; however,
p.Ser649Leu was assessed as potentially damaging due to "improper substitution in the transmembrane region". This indicates a possible involvement with the disruption of RET TMD self-association pattern by Ser-649 substitution. 


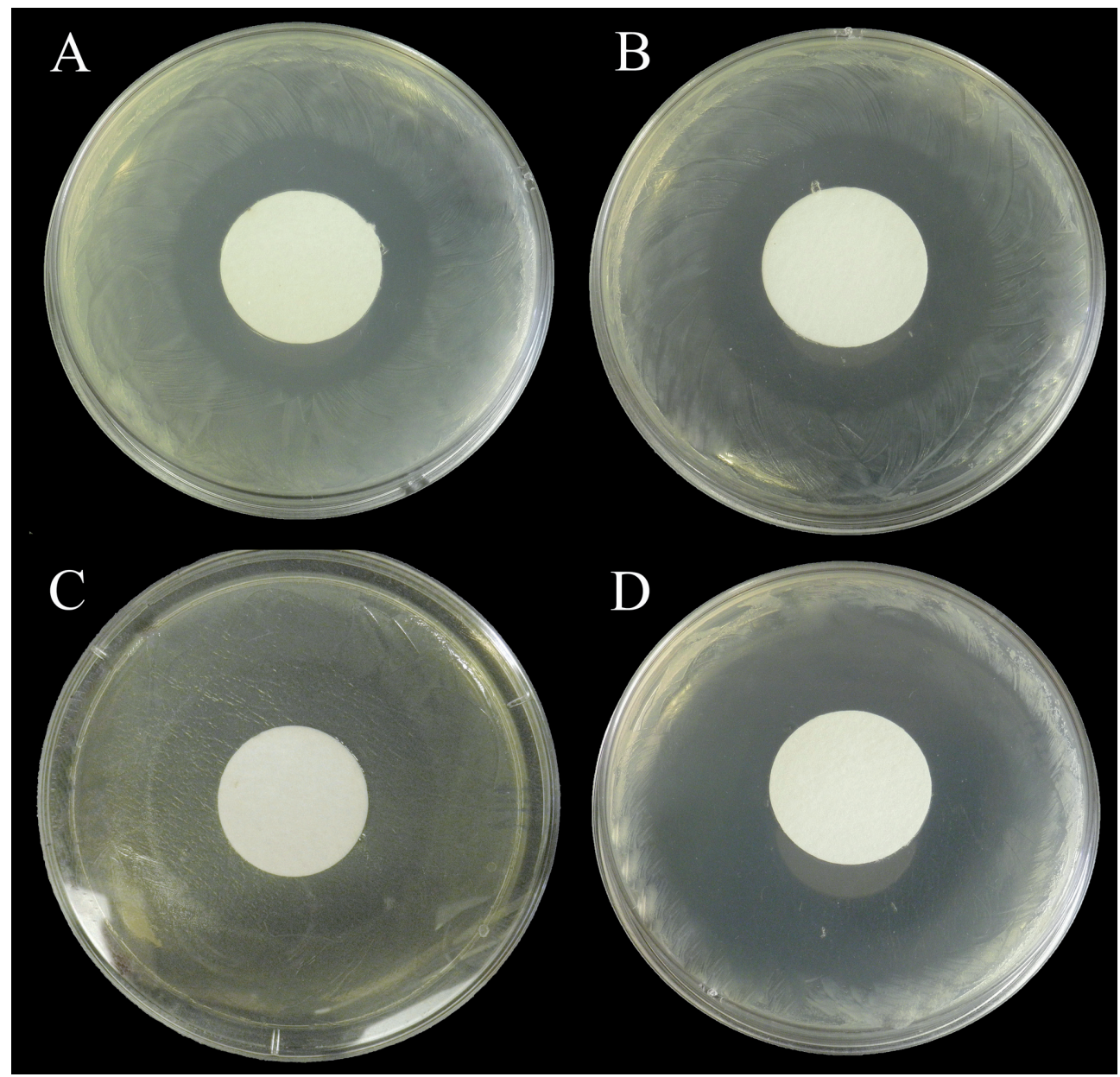

Figure 5. Disk diffusion assay. Size of the zone of inhibition (ZOI) reflects the amount of CAT protein, dependent on the extent of transmembrane domain-mediated oligomerization of the fusion proteins. Greater ZOI size reflects lesser amount of CAT protein and vice versa. Figure A) represents E.coli NT326 construct producing fusion proteins that contain RET transmembrane domain carrying p.Ser649Leu mutation. Figure B) represents E.coli NT326 construct with RET transmembrane domain carrying p.Ala641Ser mutation. Figure C) represents E.coli NT326 clone producing fusion proteins that contain RET wild-type transmembrane domain. Figure D) represents E.coli NT326 transformed with digested and religated pccKAN vector.

Validation of the fusion proteins. Both the presence and the correct topology of ToxR-RET-TMD-MBP fusion proteins were proven by their ability to grow on M9 minimal medium with maltose as the sole source of carbon. E.coli NT326 cells lack MBP protein in their periplasm and therefore are unable to utilize maltose as carbon source. Clones carrying p.Ser649Leu, p.Ala641Ser and wild-type RET transmembrane domain, respectively, proved the ability to utilize maltose as the sole source of carbon (Figure 3). Positive controls carrying GpA-WT and G83I TMD grew on the same media (data not shown). Due to the lack of fusion proteins, neither untransformed E.coli NT326 nor E.coli NT326 transformed with digested and re-ligated pccKAN plasmid grew on this medium. The viability of all the clones and controls was proved on M9 minimal medium containing glucose.
Qualitative measurement of helix-helix interaction. Qualitative evidence of transmembrane helix dimerization was reported by the rate of reporter protein-mediated chloramphenicol (CAM) resistance using the disk diffusion assay. Due to the bacteriostatic effect of CAM, a zone of growth inhibition (ZOI) circumscribing the disk with antibiotic was created (Figure 4). The size of the ZOI is in indirect proportion to the level of CAT protein, which confers resistance to CAM. Relative activities of CAT protein were compared against untransformed E.coli NT326 competent bacteria. TOXCAT clones showed various responses to chloramphenicol exposure. Analysis of triplicates measured in 10 different diameter positions leads to the following average ZOI data. For E.coli NT326 cells expressing ToxR-RETwt-MBP, the ZOI diameter was $4.71 \mathrm{~cm}$; for bacteria expressing ToxR-Ala641Ser-MBP the diameter 

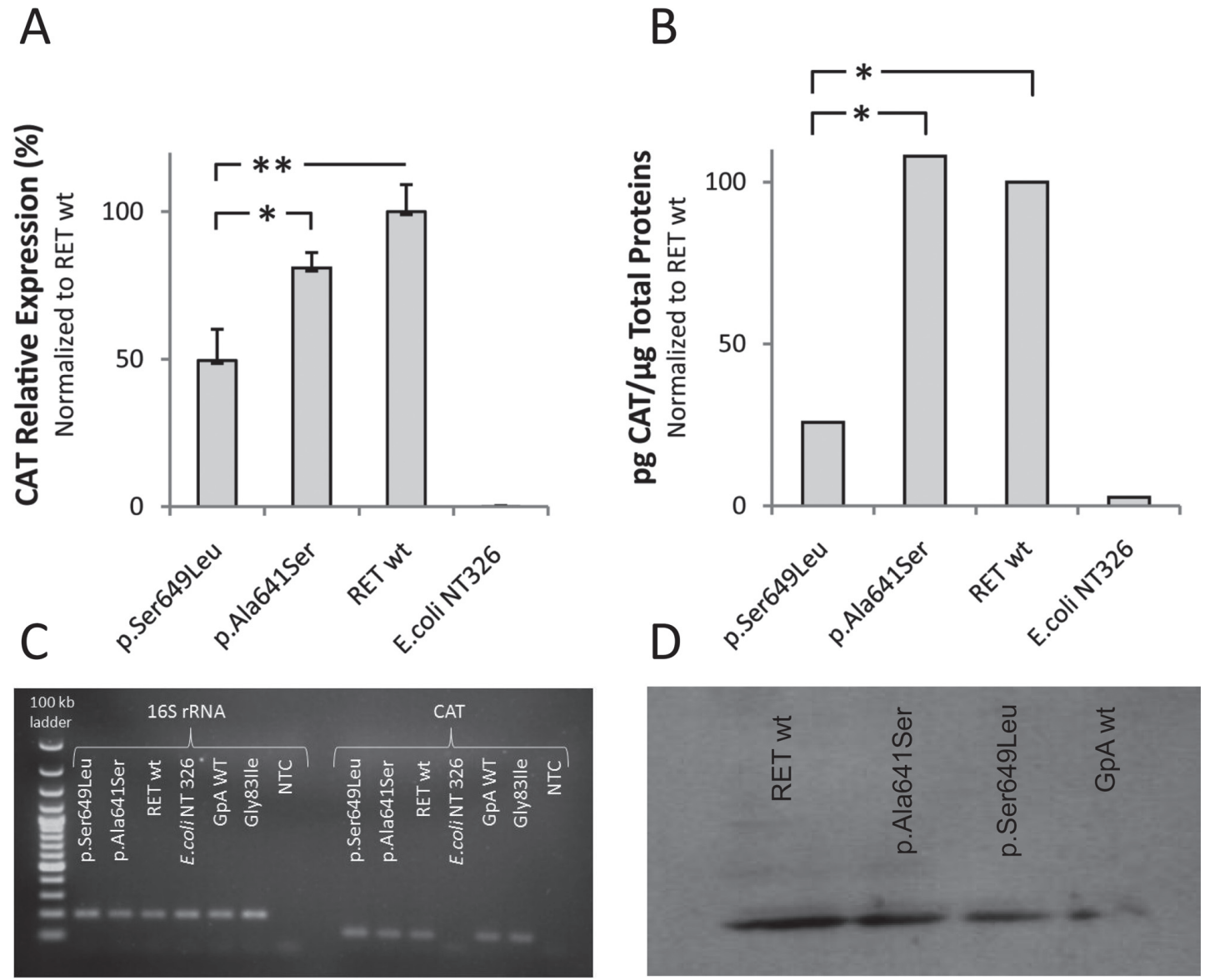

Figure 6. Quantitation of CAT reporter gene expression. Panels (A) and (C) reflect mRNA levels, panels (B) and (D) represent CAT protein levels. (A); relative expression of CAT reporter gene normalized to RET wild-type expression. ToxR-Ala641Ser-MBP fusion proteins showed $20 \%$ decrease of CAT gene expression. Most dramatic CAT expression change was observed in ToxR-Ser649Leu-MBP fusion proteins; almost 50\% decrease. Negative control was represented by untransformed E.coli NT326 bacteria. (B); levels of CAT protein, normalized to RET wild-type, as determined by ELISA. The results show no dramatic variations in relation to mRNA levels, the only observed difference was the relation between p.Ala641Ser and RET wild-type. However, on both levels, the difference was not confirmed as statistically significant. Panels (C) and (D) represent agarose gel visualization (C) and CAT protein qualitative evidence by immunoblot (D), respectively.

was $4.51 \mathrm{~cm}$; and for bacteria with ToxR-Ser649Leu-MBP $5.41 \mathrm{~cm}$ compared to $6.78 \mathrm{~cm}$ of E.coli NT326 transformed with digested and religated pccKAN vector (Figure 5). Implemented on CAT expression rate, the smallest ZOI diameter (ToxR-RETwt-MBP) represents the highest CAT expression, while the biggest ZOI (ToxR-Ser649Leu-MBP) reflects the lowest measured CAT expression rate of RET TMD TOXCAT constructs.

qPCR quantitation of CAT reporter gene expression. As the map of pccKAN plasmid was not available to us, we used BLAST (Basic Local Alignment Search Tool) to identify, which part of its sequence corresponds to CAT gene. The sequence 6275 - $6934 \mathrm{bp}$ of pccKAN was identified as CAT gene of Tn 9 transposon with $99 \%$ probability. CAT 2 primer pair and CAT 2 dual-labeled FAM probe were designed by us according to this region (Table 1). CAT reporter gene ex- pression on mRNA level was analyzed by quantitative PCR using $16 \mathrm{~S}$ rRNA gene as a housekeeping gene. As described above, reporter gene expression levels are relative to the strength of TMD self-association. According to normalized CAT expression levels, substitution p.Ala641Ser results in $19.11 \%(+/-5.27 \%$ SD) decrease of RET TMD self-association compared to that of RET wild-type. Substitution p.Ser649Leu decreases the self-associating ability by $50.46 \%+/-10.58 \%$ SD (Figure 6A). The observed variations were evaluated for statistical significance using Student's T-test. Based on the $p$-value ( $p=0.08)$, $p$.Ala641Ser substitution does not change strength of the dimerization significantly from the RET wildtype. On the other hand, the difference between p.Ser649Leu and RET wild-type $(\mathrm{p}=0.005)$, as well as between $\mathrm{p}$.Ser649Leu and $\mathrm{p}$.Ala641Ser $(\mathrm{p}=0.02)$ was statistically significant. Overall data were analyzed by ANOVA statistical model; F value 
(21.95901) was significantly higher than F-critical value (5.143253) and in accord with the overall p-value (0.001737), the data can be considered as statistically significant.

To validate the newly established method, CAT gene expression results for Glycophorin A wild-type (GpA WT) and Gly83Ile mutation described in the original TOXCAT article [15] were analyzed as well. Gly83Ile mutation dramatically reduced TMD self-association, manifested by reduced reporter gene expression, which correlated with the published results [15].

Quantitation of CAT reporter protein. On the protein level, CAT was quantified using CAT ELISA Kit (Roche) as described by Kjaer et al. [13]. Values of CAT protein for each sample were normalized to the value of RET wild-type sample (Figure 6B). In this assay, p.Ala641Ser was manifested by an increase in the CAT activity, suggesting an increase of the strength of self-association by $8.01 \%$. In case of p.Ser649Leu mutant a decrease by $74.18 \%$ was observed. Statistical significance of the observed variations was evaluated by Student's T-test. The difference between self-association of p.Ser649Leu and RET wild-type is statistically significant $(\mathrm{p}=0.047)$. As observed previously on mRNA level, the difference of p.Ala641Ser substitution and RET wild-type was not statistically significant $(\mathrm{p}=0.66)$.

\section{Discussion}

The focus of the study was placed on investigating suspected mutation-induced changes in the strength of RET TMD self-association. Due to their rare occurrence, mutations of the RET TMD associated with MTC have not been well explored and the mechanism, by which they activate $c$-RET oncogene is unclear. According to ARUP Scientific Resource for Research and Education MEN 2 database, both RET TMD mutation variants investigated in this study, p.Ala641Ser, as well as p.Ser649Leu are listed as variants of unknown significance, with unknown classification and MEN2 phenotype [14]. Yet, for therapeutic purposes, the knowledge of exact genotype: phenotype correlation is crucial. In this manner, cooperation between physicians and molecular biologists and/or geneticists is of utmost importance in order to shed more light into present understanding of the mechanisms responsible for MTC onset in these cases.

Recently, a study evaluating the performance of on-line mutation prediction tools has been published [25]. However, only two out of five in silico prediction tools consider p.Ser649Leu as pathogenic or probably damaging mutation. Nevertheless, p.Ser649Leu variant is known to be a MEN2 causative mutation resulting in a medium aggressive MTC [18]. In case of p.Ala641Ser, one out of five in silico tools predicted it as possibly pathogenic [25]. Association with MTC aggressiveness is harbored in ATA classification; mutations of the RET TMD are listed as "low risk", i.e. class A category [11]. The contribution of transmembrane domain mutation to the final aggressiveness of the disease phenotype in these cases has not been elucidated. A study published by Kjaer et al. [13] reported a possible role of RET TMD in the receptor dimerization and identified a pattern of three amino acid residues (Ala-641, Ser-649 and Ser-653) responsible for the TMD self-associating ability. The key to this ability lies in exact topology of the hydrophobic transmembrane alpha helix and in the biochemical properties of two serine residues, Ser-649 and Ser-653, in concert with the position of the alanine residue on codon 641. In this manner, RET TMD can significantly contribute to $c$-RET oncogene activation by creating transient RET homodimers which can be covalently linked by disulfide bonds between unpaired cysteine residues in the cysteine-rich domain of RET receptor.

Investigating any changes in the self-association of RET TMD caused by substitution of one of the three key amino residues may provide evidence required for better understanding of MTC mechanism of onset in case of patients with RET TMD mutations and possibly reveal the missing evidence for genotype: phenotype correlation knowledge.

p.Ala641Ser does not significantly affect RET TMD self-association. According to Kjaer's previous investigations, the nature of the substituent amino acid on codon 641 bears the potential to alter the helix topology and thus affect RET TMD self-association [13]. Serine, with its uncharged polar side chains apparently successfully substitutes the original cysteine; furthermore, the substitution creates four serine residues at the same side of the alpha helix: Ser-641, Ser-645, Ser-649 and Ser-653. This constellation of serine residues could potentially enhance the strength of self-association. Self-association data obtained from ToxR-A641S-MBP fusion proteins vary from $19.11 \%$ decrease on mRNA level to $8.01 \%$ increase on protein level relative to ToxR-RETwt-MBP proteins. However, these discrepancies were not validated as statistically significant, both on mRNA and protein level. Applied to RET TMD, the substitution of Ala- 641 by serine residue might not have a significant effect on the topology of the alpha helix and therefore no significant variation in the strength of self-association compared to RET wild-type TMD was observed. p.Ala641Ser substitution was reported in cis with the extracellular domain mutation p.Cys634Ser [26]. The contribution of p.Ala641Ser to the transforming potential of the dual mutation has been unknown. Our data suggest that the TMD mutation does not expand nor diminish the strong transforming potential of p.Cys634Ser but might act as wildtype RET TMD in this case. This supposition is supported by our unpublished observations of p.Ala641Ser as a single $c$-RET mutation, with no MTC developed in individuals even at the age of 60 years; along with the results of in silico on-line mutation prediction tool PolyPhen.

p.Ser649Leu causes reduction of RET TMD self-association. Ser-649 is one of the two serine residues that are the key players in RET TMD self-association [13]. Its substitution by leucine was manifested by $50 \%$ decrease of self-associating strength investigated on mRNA level and by $75 \%$ decrease on protein level. These observations are in harmony with DDA assay results, in silico predictions by PolyPhen tool, as well as with conclusions made by Kjaer et al. p.Ser649Leu substitution was reported as a sole mutation of $c$-RET gene in FMTC 
affected individuals with various penetrance. The significantly impaired ability of self-association suggests a novel, yet unknown mechanism of tyrosine kinase domain activation, possibly independent of RET homodimerization. Further experimentation is required in this subject. Theoretically, this evidence complicates the issue of previously suggested possible MEN2 treatment using lipid-soluble molecules targeted for RET homodimer disruption [13]. It is apparent that p.Ser649Leu has similar effect on RET homodimer formation and nevertheless triggers MTC onset.

Establishment of reporter gene expression analysis in TOXCAT system. Since its introduction, TOXCAT system has been used in many transmembrane domain dimerization studies [27-29]. The main advantage lies in providing the environment of a biological membrane to observe transmembrane helix behavior. The identity of TOXCAT fusion proteins is confirmed on both DNA and protein levels; maltose complementation assay also verifies the orientation of fusion proteins. Disk diffusion assay (DDA) provides qualitative evidence of the activity of the reporter system. We have also analyzed the obtained DDA data on quantitative basis, but the system was not sensitive enough to provide exact quantitation data. Quantitation of CAT protein levels was performed as described by Kjaer et al. using CAT ELISA Kit (Roche). However, we have empirically discovered that protein level data are extremely sensitive to variations in the OD of the cells prior to protein extraction. To avoid this and to confirm the obtained data, we focused on the gene expression levels. To determine the expression of CAT reporter gene, we have employed quantitative PCR, which was not previously utilized for TOXCAT system. The acquired CAT gene expression data, normalized using the $16 \mathrm{~S}$ housekeeper gene, are in concordance with the CAT protein quantitation data, and reflect the actual rate of CAT gene transcription triggered by dimerization of the TOXCAT fusion proteins. We have observed slight discrepancy between mRNA and protein levels, e.g. in the case of p.Ala641Ser mutation. However, these variations were expected, due to the difference between mRNA stability and/or degradation and the stability of CAT protein. In conclusion, both mRNA and protein level provided comparable results that support and confirm each other. Therefore, we recommend qPCR as one of the assays used for quantification of TOXCAT data.

TOXCAT system is currently one of the best solutions for the search for evidence that may contribute to assign mutations in TMDs with unknown mechanism to distinct disease phenotypes and thus to identify genotype: phenotype correlations required for classification, staging and therapy of the disease. We hope that our work will shed more light into this issue and prove the potential of TOXCAT system in basic research in the field of molecular oncology.

Acknowledgements: Authors thank for financial support: VEGA 2/0091/08 and Jan Korec Foundation. Words of our gratitude belong to Prof. Engelman, Yale for kind gift of pccKAN, E.coli NT326 bacteria and positive controls for the TOXCAT system, furthermore to MSc. Juraj Straka for graphical assistance and Jan Markus, PhD. and MSc. Sona Kontsekova for critical reading of the manuscript.

\section{References}

[1] PACINI F, CASTAGNA MG, CIPRI C, SCHLUMBERGER M. Medullary Thyroid Carcinoma. Clinical Oncology 2010; 22(6): 475-485. http://dx.doi.org/10.1016/ j.clon.2010.05.002

[2] SIPPEL RS, KUNNIMALAIYAAN M, CHEN H. Current management of medullary thyroid cancer. Oncologist 2008; 13(5): 539-547. http://dx.doi.org/10.1634/theoncologist.2007$\underline{0239}$

[3] RAUE F, FRANK-RAUE K. Update multiple endocrine neoplasia type 2. Familial Cancer 2010; 9(3): 449-457. http:// dx.doi.org/10.1007/s10689-010-9320-2

[4] KEISER HR, BEAVEN, M. A., DOPPMAN, J., WELLS, S. JR., BUJA, L. M. Sipple's syndrome: medullary thyroid carcinoma, pheochromocytoma, and parathyroid disease. Studies in a large family. NIH conference. Ann Intern Med 1973; 78(4): 561-579.

[5] CUNLIFFE WJ, HUDGSON P, FULTHORPE JJ, BLACK MM, HALL R et al. A calcitonin-secreting medullary thyroid carcinoma associated with mucosal neuromas, marfanoid features, myopathy and pigmentation. The American Journal of Medicine 1970; 48(1): 120-126. http://dx.doi.org/10.1016/ 0002-9343(70)90106-3

[6] FARNDON JR, LEIGHT GS, DILLEY WG, BAYLIN SB, SMALLRIDGE RC et al. Familial medullary-thyroid carcinoma without associated endocrinopathies - a distinct clinical entity. British Journal of Surgery 1986; 73(4): 278-281. http:// dx.doi.org/10.1002/bjs.1800730411

[7] TAKAHASHI M, RITZ J, COOPER GM. Activation of a novel human transforming gene, ret, by dna rearrangement. Cell 1985; 42(2): 581-588. http://dx.doi.org/10.1016/00928674(85)90115-1

[8] MULLIGAN LM, KWOK JBJ, HEALEY CS, ELSDON MJ, ENG C et al. Germ-line mutations of the ret protooncogene in multiple endocrine neoplasia type-2a. Nature 1993; 363(6428): 458-460. http://dx.doi.org/10.1038/363458a0

[9] ENG C. Common alleles of predisposition in endocrine neoplasia. Current Opinion in Genetics \& Development 2010; 20(3): 251-256. http://dx.doi.org/10.1016/j.gde.2010.02.004

[10] FRANK-RAUE K, RONDOT S, RAUE F. Molecular genetics and phenomics of RET mutations: Impact on prognosis of MTC. Molecular and Cellular Endocrinology 2010; 322(1-2): 2-7. http://dx.doi.org/10.1016/j.mce.2010.01.012

[11] KLOOS RT, ENG C, EVANS DB, FRANCIS GL, GAGEL RF et al. Medullary Thyroid Cancer: Management Guidelines of the American Thyroid Association. Thyroid 2009; 19(6): 565-612. http://dx.doi.org/10.1089/thy.2008.0403

[12] MACHENS A, HAUPTMANN S, DRALlE H. Modification of multiple endocrine neoplasia $2 \mathrm{~A}$ phenotype by cell membrane proximity of RET mutations in exon 10. Endocrine-Related Cancer 2009; 16(1): 171-177. http://dx.doi. org/10.1677/ERC-08-0096 
[13] KJAER S, KUROKAWA K, PERRINJAQUET M, ABRESCIA C, IBANEZ CF. Self-association of the transmembrane domain of RET underlies oncogenic activation by MEN2A mutations. Oncogene 2006; 25(53): 7086-7095. http://dx.doi. org/10.1038/sj.onc. 1209698

[14] MARGRAF RL, CROCKETT DK, KRAUTSCHEID P, SEAMONS R, CALDERON FR et al. The Multiple Endocrine Neoplasia type 2 RET proto-oncogene database: repository of MEN2-associated RET sequence variation and reference for genotype/phenotype correlations. Hum Mutat 2009; 30(4): 548-56. http://dx.doi.org/10.1002/humu.20928

[15] RUSS WP, ENGELMAN DM. TOXCAT: A measure of transmembrane helix association in a biological membrane. Proceedings of the National Academy of Sciences of the United States of America 1999; 96(3): 863-868. http://dx.doi. org/10.1073/pnas.96.3.863

[16] LANGOSCH D, BROSIG B, KOLMAR H, FRITZ HJ. Dimerisation of the glycophorin a transmembrane segment in membranes probed with the ToxR transcription activator. Journal of Molecular Biology 1996; 263(4): 525-530. http:// dx.doi.org/10.1006/jmbi.1996.0595

[17] WIENCH M, WYGODA Z, GUBALA E, WLOCH J, LISOWSKA Ket al. Estimation of Risk of Inherited Medullary Thyroid Carcinoma in Apparent Sporadic Patients. Journal of Clinical Oncology 2001; 19(5): 1374-1380.

[18] COLOMBO-BENKMANN M, LI ZP, RIEMANN B, HENGST $\mathrm{K}$, HERBST $\mathrm{H}$ et al. Characterization of the RET protooncogene transmembrane domain mutation S649L associated with nonaggressive medullary thyroid carcinoma. European Journal of Endocrinology 2008; 158(6): 811-816. http://dx.doi. org/10.1530/EJE-07-0817

[19] VACLAVIKOVA E, DVORAKOVA S, SYKOROVA V, BILEK R, DVORAKOVA K et al. RET mutation Tyr791Phe: the genetic cause of different diseases derived from neural crest. Endocrine 2009; 36(3): 419-424. http://dx.doi.org/10.1007/ $\underline{\text { s12020-009-9242-7 }}$

[20] AUSUBEL FM, BRENT R, KINGSTON RE, MOORE DD, SEIDMAN JG et al., editors. Current protocols in molecular biology. New York: John Wiley \& Sons, 1994.

[21] QUINTERO Y, POBLET M, GUILLAMÓN JM, MAS A. Quantification of the expression of reference and alcohol dehydrogenase genes of some acetic acid bacteria in different growth conditions. Journal of Applied Microbiology 2009; 106(2): 666-674. http://dx.doi.org/10.1111/j.1365-2672.2008.

[22] MACIAGG A, PEANO C, PIETRELLI A, EGLI T, DE BELLIS G, LANDINI P. In vitro transcription profiling of the $\sigma S$ subunit of bacterial RNA polymerase: re-definition of the $\sigma \mathrm{S}$ regulon and identification of $\sigma S$-specific promoter sequence elements. Nucleic Acids Research 2011; Jul; 39(13): 5338-5355. http:// dx.doi.org/10.1093/nar/gkr129

[23] LANE DJ. 16S/23S rRNA sequencing. In: Stackebrandt E, Goodfellow M, editors. Nucleic acid techniques in bacterial systematics. New York: John Wiley \& Sons, 1991: 115-175.

[24] RAMENSKY V, BORK P, SUNYAEV S. Human non-synonymous SNPs: server and survey. Nucleic Acids Research 2002; 30(17): 3894-3900. http://dx.doi.org/10.1093/nar/ gkf493

[25] CROCKETT DK, PICCOLO SR, RIDGE PG, MARGRAF RL, LYON E et al. Predicting Phenotypic Severity of Uncertain Gene Variants in the RET Proto-Oncogene. PLoS ONE 2011; 6(3): e18380. http://dx.doi.org/10.1371/journal. pone. 0018380

[26] POTURNAJOVA M, ALTANEROVA V, KOSTALOVA L, BREZA J, ALTANER C. Novel germline mutation in the transmembrane region of RET gene close to Cys634Ser mutation associated with MEN 2A syndrome. Journal of Molecular Medicine-Jmm 2005; 83(4): 287-295. http://dx.doi. org/10.1007/s00109-004-0604-6

[27] DUONG MT, JASZEWSKI TM, FLEMING KG, MACKENZIE KR. Changes in apparent free energy of helix-helix dimerization in a biological membrane due to point mutations. Journal of Molecular Biology 2007; 371(2): 422-434. http://dx.doi. org/10.1016/j.jmb.2007.05.026

[28] OATES J, KING G, DIXON AM. Strong oligomerization behavior of PDGF beta receptor transmembrane domain and its regulation by the juxtamembrane regions. Biochimica Et Biophysica Acta-Biomembranes 2010; 1798(3): 605-615. http://dx.doi.org/10.1016/j.bbamem.2009.12.016

[29] STOKES KD, RAO AG. The role of individual amino acids in the dimerization of CR4 and ACR4 transmembrane domains. Archives of Biochemistry and Biophysics 2010; 502(2): 104-111. http://dx.doi.org/10.1016/j.abb.2010.07.018 